Approved for public release: distribution is unlimited

Title:

\title{
Landscapes and Dynamics of Proteins
}

Author(s):
Toel Anderson

Stefan Boettcher

Mark Buchanan

Kelvin Chu

Chuali Chang

Richard Dyer

Doyne J. Farmer

Hans Frauenfelder

Klaus-Dieter Fritsch

Angel Garcia

Robert Gennis

Klaus Gerwert

Maximo Granados

Lydia Gregoret

Peter Hanggi

Bai-lin Hao

Gerhard Hummer

Eric Jeckelmann

Ioannis Kevrekidis

Janos Ladik

Gustavo Lopez

Ben McMahon

M. Muthukumar

Ulrich G. Nienhaus

Robert Nigmatulin

Eric Olson

Pal Ormos

Graham Palmer
University of Washington

LANL, P-21

Emory University

Nature Magazine

University of Vermont

University of California, Irvine

LANL, CST-4

The Prediction Company

LANL, CNLS, P.I.*

University of Bayreuth

LANL, T-10

Univ Ill at Urbana- Champaign

Ruhl Univ, Bochum, Germany

Univ Autonoma de Zacatecas

Univ of California, Santa Cruz

University of Augsburg

Chinese Academy of Sciences

LANL, T-10

University of California, Irvine

Princeton University

Univ of Erlangen-Nuremberg

Univ of Guadalajara, Mexico

LANL, CNLS

University of Massachusetts

University of Ulm, Germany

Rensselaer Polytechnic Institute Indiana University

Biophys Inst, Szeged, Hungary

Rice University

Los Alamos National Laboratory, an affirmative action/equal opportunity employer, is operated by the University of California for the U.S. Department of Energy under contract W-7405-ENG-36. By acceptance of this article, the publisher recognizes that the U.S. Govemment retains a nonexclusive, royaltyfree license to publish or reproduce the published form of this contribution, or to allow others to do so, for U.S. Government purposes. Los Alamos National free license to publish or reproduce the published form of this contribution, or to allow o wersices of the U.S. Department of Energy. Los Alamos National Laboratory strongly supports academic freedom and a researcher's right to publish; as an institution, however, the Laboratory does not endorse the

viewpoint of a publication or guarantee its technical correctness. 
Fritz Parak

Matteo Pellegrini

Alan Perelson

Michel Peyrard

Cong-Kha Pham

George Pimbley

David Pines

Anders Sandvik

Rudolf Rigler

Michael Schick

Joerg Schmalian

Gunther Schoeck

Alfons Schulte

Solomon Stavrov

Daan Thorn-Leeson

Zoltan Toroczka

Peter Wolynes

William Woodruff
Tech Univ, Munich, Germany Univ of Calif, Los Angeles

LANL, T-10

Ecole Normale Superi de Lyon

Tokyo University

Self-employed

Univ Ill at Urbana-Champaign

University of Illinois

Karolinska, Institute

University of Washington

University of Illinois

University of Vienna

University of Central Florida

Tel Aviv University

LANL, CNLS

VA Polytech Inst./State Univ Univ Ill at Urbana-Champaign

LANL, CST-4

Submitted to: DOE Office of Scientific and Technical Information (OSTI)

\section{Los Alamos}

NATION LL L I BORATORY

Los Alamos National Laboratory, an affirmative action/equal opportunity employer, is operated by the University of Catifornia for the U.S. Department of Energy under contract W-7405-ENG-36. By acceptance of this article, the publisher recognizes that the U.S. Government retains a nonexclusive, royaltyfree license to publish or reproduce the published form of this contribution, or to allow others to do so. for U.S. Government purposes. Los Alamos National Laboratory requests that the publisher identify this article as work pertormed under the auspices of the U.S. Department of Energy. Los Alamos National Laboratory strongly supports academic freedom and a researcher's right to publish; as an institution, however, the Laboratory does not endorse the viewpoint of a publication or guarantee its technical correctness. 


\section{Landscapes and Dynamics of Proteins}

\section{Authors}

Niels Anderson $\checkmark$ Joel Berendzen $r$ Stefan Boettcher $v$ Mark Buchanan $r$ Kelvin Chu ${ }^{2}$ Chuali Chang ${ }^{\prime}$ Richard Dyer Doyne J. Farmer $\checkmark$ Hans Frauenfelder $\checkmark$ Klaus-Dieter Fritsch $\mathcal{C}$ Angel Garcia Robert Gennis Klaus Gerwert Maximo Granados 2 Lydia Gregoret $\checkmark$ Peter Hanggi $\checkmark$ Bai-lin $\mathrm{HaO} v$ Gerhard Hummer $\checkmark$ Eric Jeckelmann $v$ Ioannis Kevrekidis $\checkmark$ Janos Ladik $v$ Gustavo Lopez $\checkmark$ Ben McMahon $\checkmark$ M. Muthukumar $\checkmark$ Ulrich G. Nienhaus Robert Nigmatulin Eric Olson Pal Ormos Graham Palmer Fritz Parak Matteo Pellegrini Alan Perelson Michel Peyrard Cong-Kha Pham George Pimbley David Pines Anders Sandvik Rudolf Rigler Michael Schick Joerg Schmalian Gunther Schoeck Alfons Schulte Solomon Stavrov Daan Thorn-Leeson Zoltan Toroczka Peter Wolynes William Woodruff
University of Washington

LANL, P-21

Emory University

Nature Magazine

University of Vermont

University of California, Irvine

LANL, CST-4

The Prediction Company

LANL, CNLS,*(e-mail: frauenfelder@lanl.gov)

University of Bayreuth

LANL, T-10

University of Illinois at Urbana-Champaign

Ruhl University, Bochum, Germany

University Autonoma de Zacatecas

University of California, Santa Cruz

University of Augsburg

Chinese Academy of Sciences

LANL, T-10

University of California, Irvine

Princeton University

University of Erlangen-Nuremberg

University of Guadalajara, Mexico

LANL, CNLS

University of Massachusetts

University of Ulm, Germany

Rensselaer Polytechnic Institute

Indiana University

Bisophysics Institute, Szeged, Hungary

Rice University

Technical University, Munich, Germany

University of California, Los Angeles

LANL, T-10 $\checkmark$

Ecole Normale Superieure de Lyon

Tokyo University

Self-employed

University of Illinois at Urbana-Champaign

University of Illinois

Karolinska, Institute

University of Washington

University of Illinois

University of Vienna

University of Central Florida

Tel Aviv University

LANL, CNLS

Virginia Polytechnic Inst. \& state University University of Illinois at Urbana-Champaign

LANL, CST-4 


\begin{abstract}
This is the final report of a three-year, Laboratory Directed Research and Development (LDRD) project at Los Alamos National Laboratory (LANL). Complex systems have become a major focus for research in physics, chemistry, and materials science. Four aspects are essential for understanding complex systems: structure, energy landscape, dynamics, and function. Proteins, the building blocks of living systems, are ideal for studying the properties and laws of complex systems. We have been exploring heme proteins, in particular myoglobin, experimentally, theoretically, and computationally. We have arrived at a model that combines structure, energy landscape, dynamics, and function for an apparently simple biological process: the binding of a small molecule to a protein. The model can most likely be generalized to many other proteins in general and to other complex systems. The understanding may lead to novel proteins, novel materials, and possibly also to new insight into problems such as biological threats. The enormous complexity of biological systems means that the extension to other systems is not a short-term endeavor, but must be continued for a considerable time to reap the full benefit of such studies.
\end{abstract}

\title{
Background and Research Objectives
}

Complex systems, from glasses to biomolecules and the immune system, all appear to share one major property: Their ground state is highly degenerate and must be described by an energy landscape (EL). The more complex the system, the more involved is the EL. In "simple" systems, from atoms to nuclei, and crystalline solids, studies of the energy levels have been crucial for a full understanding of the physics. We expect that similar progress will be made on complex systems by studying the EL. At present, however, even in the least complex systems such as glasses and spin glasses, the EL is not fully known. Even less is known about the laws that govern the dynamics in complex systems, in other words, the motions in the hyperspace of the EL. Some general approximate laws have been found, but a deep understanding is still lacking. The goal of this work is to investigate, in close contact with experiment, the EL and the relevant reaction both theoretically and computationally.

In addition to energy landscapes we also propose to study various types of fitness landscapes. One type of fitness landscape is the affinity landscape where to each antibody

*Principal Investigator, e-mail: frauenfelder@lanl.gov 
gene sequence an affinity for a particular ligand is assigned. Equivalently, the antibody can be fixed and a set of ligands with differing sequences examined. In either case, depending upon the rule of assigning affinity to sequence, different landscapes result. We will examine the properties of different landscapes such as the number of local optima and the statistical properties of move-rules on a landscape. For instance, if only uphill moves are allowed, what is the probability distribution for the number of mutations until one gets trapped at a local optimum? How does the dynamics change if the landscape has large flat regions that arise due to the many-to-one nature of the mapping between sequence and structure? We shall also examine the effects of correlation among the affinities of different sequences on the ability to search sequence space for high-affinity molecules. Such searches can be conducted in the laboratory by a biotechnology company or in vivo by the immune system during the course of affinity maturation. We believe that elucidating the search strategies employed by the immune system may give insights into strategies that biotechnology companies can exploit.

The structure of a molecule influences its fitness. An interesting example of this effect is seen in RNA viruses. If we view the rate of replication of the virus as its fitness, then one can show that the fitness depends on the structure of the RNA molecule. We can begin to model this effect by using RNA secondary-structure-prediction algorithms to assign a secondary structure to each sequence, and then assign a fitness based on the degree of base pairing in the structure. We can then ask questions about the evolution of the viral sequence based on mutation rate and rules for fitness based-selection.

Applied molecular evolution, or molecular diversity, is a general new approach to the discovery of useful molecules and to the analysis of structure-function relationships in such molecules. Applications range from discovery of new pharmaceuticals, to biosensors, to the generation of materials with novel properties. The central idea is to generate vast libraries for potentially useful molecules. Thereafter, successive rounds of mutation, recombination, and selection are employed to yield increasingly improved variants in a manner somewhat akin to natural evolutionary processes. The "landscape" of the property of interest, e.g. binding affinity, is therefore sampled at a variety of points in a structured way. 
The research objectives follow logically from the problems just sketched. In a few selected systems, structure, energy landscape, dynamics, and function must be studied in detail and the connections must be established. At first, this path appears to be straightforward. The work performed under this LDRD project and research performed during the past 25 years proves, however, that even apparently simple biological processes are sophisticated and beautifully executed.

\section{Importance to LANL's Science and Technology Base and National R\&D Needs}

Our work contributes directly to two DOE Competencies:

Advanced Computing, Modeling, and Simulation

Bioscience and Biotechnology.

More specifically, the work performed during the past three years and the work that we hope to perform in the future can have the following impact:

1. It will help establish a science base for work on biological threats.

2. At present, most of the work in the biosciences and biotechnology is done with limited input from the physical and computational sciences. Our research constructs a rigorous base for the design and function of biopolymers.

3. Young scientists working on the interface between physics, chemistry, computing, and biology, as in our research, will form the cadre for the future DOE efforts in bioscience and biotechnology.

4. The DOE has started a new initiative on "Complex and Collective Phenomena". Our work, and related efforts in other groups at LANL, will give us a head start in contributing to this initiative.

5. The biosciences and biotechnology will be an increasingly important part of the work at LANL, from efforts on bio-threats, to structural and functional genomics. At present, much of this work at LANL and in other laboratories is done without strong interdisciplinary connections linking biology, chemistry, computing, and physics. Our work is fully integrated and can be a model for future major initiatives. 


\section{Scientific Approaches and Accomplishments}

We describe here the major accomplishments. Where the results have been published, we will be brief. We provide details only on work that has not yet been published. The accomplishments fall into three categories, research, workshops and conferences, and lectures.

Research-The Approach. (For details see Frauenfelder, LA-UR 95-4357. The Complexity of Proteins.) Myoglobin ( $\mathrm{Mb}$ ), our "hydrogen atom of biology", is a moderately sized protein that contains a heme group with a central iron atom. This central iron atom binds dioxygen and carbon monoxide. In the simplest version, the reaction with $\mathrm{CO}$ can be written as

$$
\mathrm{Mb}+\mathrm{CO} \rightarrow \mathrm{MbCO}
$$

Initially, this reaction was considered to be a one-step process. Our work, starting before 1975, showed that the process is far more complicated. ${ }^{1}$ By performing experiments over a wide range of time and temperature (from about 10 to $300 \mathrm{~K}$ ) we found that the reaction occurs in a series of steps. Despite hundreds of experimental, computational, and theoretical publications, the process has not been fully understood until now.

The approach to study the $\mathrm{Mb}$ reaction is in principle very simple. $\mathrm{MbCO}$ is placed into a cryostat. A short laser pulse breaks the $\mathrm{Mb}-\mathrm{CO}$ bond and $\mathrm{CO}$ moves away from the iron. The subsequent rebinding, $\mathrm{Mb}+\mathrm{CO} \rightarrow \mathrm{MbCO}$ is observed optically. The reaction pathway is deduced from the time and temperature dependence of this process.

Why is this reaction important? How is the problem related to the energy landscape and dynamics? What can we learn that is relevant for other proteins and other complex systems? What could the impact of a complete solution be for bioscience and biotechnology? The answers to these questions are not fully known, but some aspects are already clear.

Research - Importance. At present, the function of not even one protein is understood quantitatively. Understanding one protein fully would help understand others and possibly lead to the construction of mutated proteins with desired properties, a goal important for biomaterials, medicine, and pharmacology. If myoglobin were a member of an unimportant 
class, a complete understanding would not be too exciting. $\mathrm{Mb}$, however, is a member of the hemoglobin family. ${ }^{2}$ Figure 1, copied from Hardison ${ }^{2}$, shows that the hemoglobins appear extremely widely and can have very different functions. Understanding Mb quantitatively can be the key to many secrets.

Research - Structure, Energy Landscape, Dynamics, and Function. For many years, proteins were considered to be nearly static structures. While a few papers pointed to the importance of motions, the beautiful structures obtained by x-ray diffraction gave the impression of well-ordered systems, with a unique structure and a well-defined energy. The work in Ref. 1 proved that this impression was wrong; a given protein can assume a very large number of somewhat different conformations, called conformational substates (CS). ${ }^{3}$ The energy landscape describes the connection between protein structure and energy. Protein motions (protein dynamics) are described as jumps between various CS. The goal of our work is to connect structure, energy landscape, dynamics, and function for the case of the binding of $\mathrm{CO}$ to $\mathrm{Mb}$. The problems to be solved are the following. After photodissociation, the $\mathrm{CO}$ leaves the $\mathrm{Mb}$ with a high probability. When $\mathrm{CO}$ tries to enter again, it has to compete with the water molecules that surround the protein. How can it compete effectively? What are the protein motions that are involved? A complete answer to these questions will be given in a paper in preparation, but some of the essential features are sketched here.

A major step towards the goal of connecting structure and function was made by the work of Joel Berendzen and his collaborators. ${ }^{4}$ They measured the structure of the Mb-CO complex at low temperature before and after photolysis Figure 2 shows the region around the heme in $\mathrm{Mb}$. In Figure $2 \mathrm{a}$, the $\mathrm{CO}$ is bound to the heme iron; in Figure 2b, the $\mathrm{CO}$ sits in the heme pocket parallel to the heme. Figure 2 also shows the existence of two pockets, space not occupied by atoms of the protein. The so-called heme pocket is on the side where the $\mathrm{CO}$ is bound (distalside), the so-called xenon pocket is on the opposite (proximal) side. 
Both pockets are formed mainly by residues that have been conserved in the course of evolution.

The exit and entry of $\mathrm{CO}$ can now be described as follows. Immediately after photodissociation, the heme iron moves partially out of the heme plane, making rebinding of the $\mathrm{CO}$ difficult. For a few femtoseconds, the $\mathrm{CO}$ ricochets around the heme pocket and then docks at the site shown in Figure 2b. After about 100 nanseconds, it moves out into the solvent, either directly or through the xenon pocket. When there is no $\mathrm{CO}$ in the heme pocket, a water molecule can move in. Because there are many more water than $\mathrm{CO}$ molecules around the protein, the entrance of water is much more likely and water thus can block the direct reentrance of the $\mathrm{CO}$ into the heme pocket. The $\mathrm{CO}$ therefore moves to the xenon pocket and waits there until the water moves out of the heme pocket. It then transits to the distal side and binds. This scenario connects structure, energy landscape, dynamics, and function. The quantitative description in terms of these features is, of course, more intricate and we are in the course of writing a complete version. It is intriguing to note that xenon pockets have been found in many other proteins. Do these have an important, as yet unrecognized, function?

Workshops and Conferences. The Center for Nonlinear Studies (CNLS) organizes a large number of workshops and conferences each year. Some of the ones that were held during the past three years were directly connected with the subject of this LDRD project. These conferences establish contacts between LANL researchers and an outstanding group of U. S. and foreign scientists. At the same time, they provide visibility to the LANL research. We list here the two most important conferences.

1. Landscape Paradigms in Physics and Biology: Concepts, Structures, and Dynamics, $16^{\text {th }}$ Annual International Conference of the Center for Nonlinear Studies, Los Alamos, NM, May 13-17, 1996. This conference attracted an outstanding group of researchers from a wide range of fields. The papers from the conference appeared in Physica D 107, 117-435 (1997) and are collected in a volume edited by H. Frauenfelder, A. R. Bishop, A. Garcia, A. Perelson, P. Schuster, D. Sherrington, and P. J. Swart and published by Elsevier in 1997. 
2. Third International Symposium on Biological Physics, Santa Fe, NM, September 20-24, 1998. This symposium, organized mainly by CNLS, was very well attended. A volume is in preparation.

Lecture Series and Lectures. A lecture series on "Biology for Physicists" exposed many of the LANL physicists to the basic biological concepts. In addition, Hans Frauenfelder presented a large number of lectures, describing the CNLS work, both at national and international conferences.

\section{Publications}

1. Frauenfelder, H. "Proteins and the Physics of Complexity," in Nonlinear Excitation in Biomolecules, Pp 177-189, M. Peyrard, Ed., Springer-Verlag, Berlin (1995).

2. Frauenfelder, H. "Komplexitat in der belebten Materie" Schriftenrcihe Ernst-AbbeKolloquium Jena. 9, 1-26 (1995).

3. Frauenfelder, H. "Proteins: A Challenging Many Body Problem," Phil. Mag. B74, 579-585 (1996).

4. Johnson, J. B., Lamb, D. C., Frauenfelder, H., Muller, J. D., McMahon, Ben, Nienhaus, G. U. and Young, R. D. "Ligand Binding to Heme Proteins VI: Interconversion of Taxonomic Substates in Carbomonoxymyoglobin," Biophys. J. 71, 1563-1573.

5. Frauenfelder, H. "The Complexity of Proteins, In Physics of Biological Systems-From Molecules to Species," Eds. H. Flyvbjerg, J. Hertz, M. H. Jensen, O. G. Mouritse, and K. Sneppen, Springer-Verlag, Verlin, Pp. 29-60 (1997).

6. Frauenfelder, H. "Proteins as Paradigms of Complex Systems," in Rontgen Centennial, Eds. A. Haasc, G. Landwehr, and F. Umbach, World Scientific, Singapore, Pg. 227-241 (1997).

7. Frauenfelder, H. and Wolynes, P. G., "Picturing the Working Protein," in Theoretical and Computational Genome Research, S. Suhai, Ed., Plenum Press, New York, Pp 231236 (1997).

8. Frauenfelder, H. "Proteins and Glasses," in Structure and Dynamics of Glasses and Glass Formers, C. A. Angell, K. L. Ngai, K. Kieffer, T. Egami, and G. U. Nienhaus, Eds., Materials Research Society, Pittsburgh, Pp. 343-347, (1997). 
9. Garcia, A. F., Krumhansl, J. A. and Frauenfelder, H., "Variations on a Theme by Debye and Waller: From Simple Crystals to Proteins," Proteins, 29, Pp.153-160 (1997).

10. Nienhaus, G. U., Muller, J. D. McMahon, B. H. and Frauenfelder, H. "Exploring the Conformational Energy Landscape of Proteins," Physica D 107, 297-331 (1997).

11. Frauenfelder, H. McMahon, B. Stojkovic, B. P. and Chu, K., "Is Myoglobin like a Swiss Watch?," in Nonlinear Cooperative Phenomena in Biological Systems, L. Matson, Ed., World Scientific, Singapore, pp.145-164 (1998).

12. Frauenfelder, H, "Protein Dynamics and Function," in Protein Dynamics, Function, and Design, O. Jardetzky and J. F. Lefevre, Eds; , Plenum Press, New York, pp-95-102 (1998).

13. Frauenfelder, H. and McMahon, B., "Dynamics and Function of Proteins," Proc. Natl, Acad. Sci. USA, 95, 4795-4797 (1998).

14. Frauenfelder, H and Thorn Leeson, D., "The Energy Landscape in Non-biological and Biological Molecules," Nature Structural Biology, 5, 757-759 (1998).

15. Deissler, Robert J, and Brand, Helmut R., "The Effect of Nonlinear Gradient Terms on Breathing Localized Solutions in the Quintic Complex Ginzburg-Landau Equation," Physical Review Letters, 81 pp 3856-3859 (1998).

16. Frauenfelder, H., Wolynes, P. G., and Austin, R. H., "Biological Physics," Rev. Mod. Phys., Centennial Issue 1999 (in press).

\section{References}

[1] Austin, R.H., Beeson, K., Eisensein, L., Frauenfelder, H., and Gunsalus, I.C., "Dynamics of Ligand Binding to Myoglobin," Biochemistry 14, 5355-5373 (1975).

${ }^{[2]}$ Hardison, R. "The Evolution of Hemoglobin," American Scientist 87, 126-137 (1999).

[3] Frauenfelder, H., Petsko, G.A., and Tsernoglou, D., "Temperature-Dependent X-Ray Diffraction as a Probe of Protein Structural Dynamics," Nature 280, 558-563 (1979).

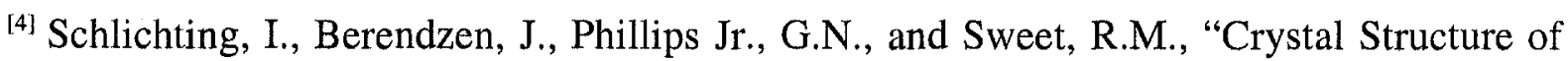
Photolysed Carbonmonoxy-Myoglobin," Nature 371, 808-812 (1994). 


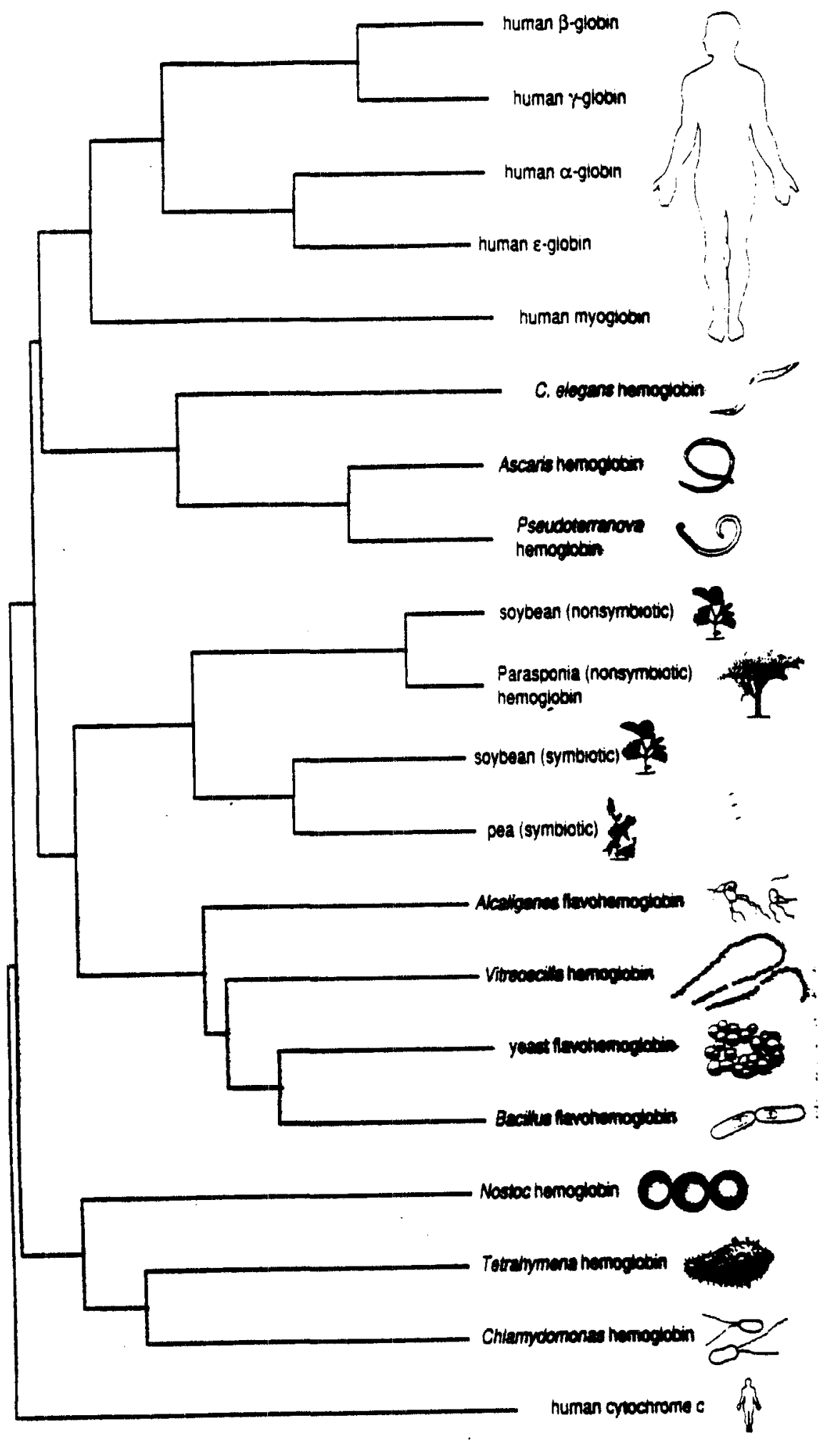

Fig. 1 The family of hemeglobins. (From Hardison, ref.2.) 


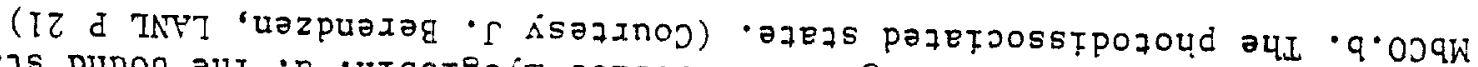

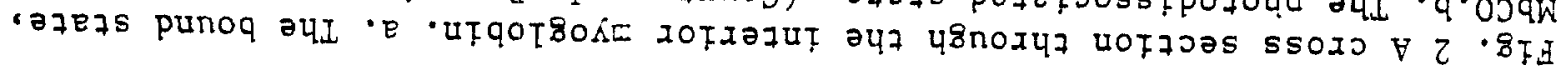

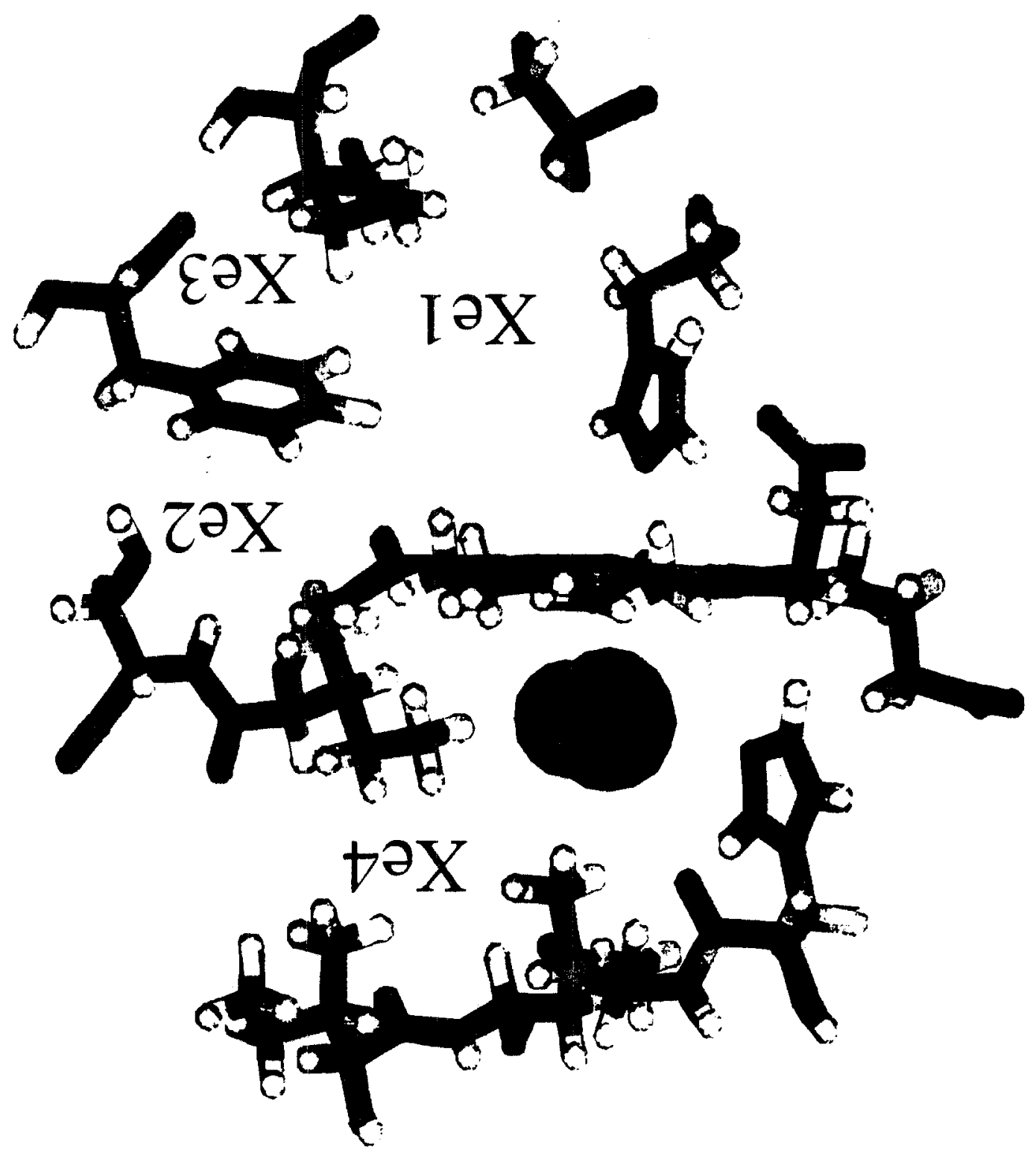

